\title{
Erythrocyte Membrane Proteins in Hereditary Glucosephosphate Isomerase Deficiency
}

\author{
T. Coetzer and S. S. ZaIL, Department of Hematology, School of Pathology, The \\ South African Institute for Medical Research, and The University of the \\ Witwatersrand, Johannesburg, South Africa
}

A B S T R A C T Erythrocytes ( $=50 \%$ reticulocytes) obtained from a splenectomized patient with a thermolabile variant of glucosephosphate isomerase (GPI) deficiency showed a striking degree of crenation and decreased filterability through $3-\mu \mathrm{m}$ Nuclepore filters (Nuclepore Corp., Pleasanton, Calif.). Membranes prepared by hypotonic lysis of such erythrocytes were found to contain a high molecular weight aggregate which was probably disulphide-bonded. The $10 \%$ most dense erythrocyte fraction showed an accentuation of aggregate formation while aggregates could not be detected in the $10 \%$ least dense erythrocyte fraction. The aggregate consisted mainly of spectrin (band 1) and a protein with the mobility of 4.2. "Extractability" of spectrin from these membranes was also markedly diminished. Incubation of the erythrocytes for $24 \mathrm{~h}$ in substrate-free medium caused more pronounced spectrin aggregation than in low or high reticulocyte controls. Incubation of low or high reticulocyte controls for $24 \mathrm{~h}$ in medium that contained glucose completely prevented the formation of the high molecular weight aggregate. GPI-deficient erythrocytes incubated with glucose in the medium showed an accentuation of membrane protein aggregate formation; however, this was almost completely reversed by the addition of adenine and inosine to the incubation medium or by the use of fructose, the intermediate just distal to the "block" in glycolysis, as the sole substrate. ATP and reduced glutathione levels in the GPIdeficient erythrocytes incubated with glucose were similar to that found in the low and high reticulocyte controls. Our findings suggest that only a proportion of erythrocytes (the older, more dense population of cells) are susceptible to the formation of disulphide-bonded aggregates, and that this is directly related to an impairment of substrate flow through the glycolytic sequence. The exact mechanism of aggregate formation in these erythrocytes remains to be elucidated.

Received for publication 4 August 1978 and in revised form 23 October 1978.

\section{INTRODUCTION}

Although a relatively rare condition, hereditary glucosephosphate isomerase (GPI) ${ }^{1}$ (D-glucose-6-phosphate ketol-isomerase, EC 5.3.1.9) deficiency is probably the third most commonly occurring erythrocyte enzymopathy (1). The underlying mechanism of hemolysis in this, as well as in other hereditary erythrocyte enzymopathies, is poorly understood, as indicated in recent reviews by Paglia and Valentine (1) and Schröter and Tillman (2). In particular, there appears to be no correlation between the degree of hemolysis and the biochemical properties of GPI-deficient erythrocytes such as the residual enzyme activity and the in vitro thermostability of GPI variants.

In a recent study, Schröter and Tillman (3) found markedly decreased deformability of young as well as whole-erythrocyte populations in four patients with different types of GPI-deficiency. The increased erythrocyte rigidity was correlated with an increased hemoglobin content in isolated membranes and with incubation of whole cells at an acid $\mathrm{pH}$. They suggested that the splenic circulation with its low $\mathrm{pH}$ impairs the deformability of GPI-deficient erythrocytes and predisposes them to splenic sequestration. In the present study we present our findings in a patient with a thermolabile GPI variant who has a high molecular weight aggregate of membrane proteins in freshly prepared erythrocyte membranes, which we speculate may contribute to decreased deformability of these erythrocytes and their premature destruction.

\section{METHODS}

Subject. The patient with GPI-deficiency has been the subject of a recent report (4). In summary (by courtesy of

${ }^{1}$ Abbreviations used in this paper: G6PD, glucose-6phosphate dehydrogenase; GPI, glucosephosphate isomerase; GSH, glutathione; PBS, phosphate-buffered saline; PK, pyruvate kinase; SDS-PAGE, polyacrylamide gel electrophoresis in sodium dodecyl sulphate. 
Dr. E. Cayanis), she is a 10-yr-old Black South African child, who was splenectomized in 1970, and has a hemoglobin value ranging between 8.5 and $10 \mathrm{~g} / 100 \mathrm{ml}$ and a reticulocyte count ranging from 32 to $71 \%$. She is on folic acid supplementation. GPI activity in her erythrocytes was very low (3.5-5.3 $\mathrm{U} / \mathrm{g}$ hemoglobin), as compared with values of $10.8-18.2 \mathrm{U} / \mathrm{g}$ hemoglobin in seven heterozygotes and values of 29.0 and 37.8 $\mathrm{U} / \mathrm{g}$ hemoglobin in two unaffected members of her family. The range in a control population was $21.5-35.3 \mathrm{U} / \mathrm{g}$ hemoglobin. Electrophoretic mobility and pH optima of GPI in the patient's hemolysates were normal, but the enzyme was thermolabile, retaining only $7 \%$ of its activity when incubated for $5 \mathrm{~min}$ at $54^{\circ} \mathrm{C}$. Michaelis constants for glucose-6-phosphate and fructose-6-phosphate, and inhibitor constants for 6phosphogluconate with respect to glucose-6-phosphate and fructose-6-phosphate as substrates, were all similar to the normal wild-type enzyme. Of some importance to the studies reported below, the patient's ATP levels were raised $(1.83 \mathrm{mmol} /$ liter packed erythrocytes) compared with mean control values $( \pm 1$ SD) of $1.05 \pm 0.15$ mmoles/liter packed erythrocytes. ATP stability as determined by Oski and Naiman (5) was, however, impaired, there being a $16 \%$ decrease compared with mean control values $( \pm 1$ SD) of $9.7 \pm 1.4 \%$. This probably reflects, at least in part, decreased ATP stability in a youngcell population (5).

In addition to control subjects who consisted of healthy laboratory personnel, three other subjects were used as "high reticulocyte" controls. One was a splenectomized boy of 11 yr with pyruvate kinase deficiency (hemoglobin, $9.5 \mathrm{~g} / 100 \mathrm{ml}$; reticulocytes, 40-51\%), and another was an 18-yr-old nonsplenectomized White male with severe glucose-6-phosphate dehydrogenase deficiency (as yet uncharacterized) (hemoglobin, $13.5 \mathrm{~g} / 100 \mathrm{ml}$; reticulocytes, $35 \%$ ). The third subject was a 56-yr-old female with idiopathic autoimmune hemolytic anemia (hemoglobin, $7.4 \mathrm{~g} / 100 \mathrm{ml}$; reticulocytes, $46 \%$ ).

Preparation of erythrocyte membranes. Heparinized venous blood was obtained from the patient or controls and immediately cooled in an ice bath. The cells were washed four times in $10 \mathrm{vol}$ of $0.9 \% \mathrm{NaCl}$ at $4^{\circ} \mathrm{C}$, care being taken to remove the residual buffy coat as completely as possible after each centrifugation. Erythrocyte ghosts were prepared by hypotonic lysis in ice-cold 20 mosmol Tris buffer, pH 7.6, which contained $1 \mathrm{mM}$ EDTA, and were washed four times in $20 \mathrm{vol}$ of this buffer after centrifugation at $30,000 \mathrm{~g}$ for 15 min. Almost-white ghosts were obtained. The addition of EDTA to the lysing buffer was found to be essential as it inhibits cross-linking but does not reverse it (6-8). The ghosts were solubilized in 1\% SDS as described by Fairbanks et al. (9), except that mercaptoethanol was used in place of dithiothreitol in the solubilizing solution. Duplicate membrane preparations were also solubilized in the absence of reducing agent.

Polyacrylamide gel electrophoresis in sodium dodecyl sulphate (SDS-PAGE). The membrane polypeptides were separated and stained with Coomassie Blue (Bio-Rad Laboratories, Richmond, Calif.) in one-dimensional separations after electrophoresis in $3 \%$ polyacrylamide gels containing $0.1 \%$ SDS as described by Fairbanks et al. (9). $50 \mu \mathrm{g}$ of membrane protein were loaded on all gels, except in the studies of spectrin "extractability" (vide infra), when only $10 \mu \mathrm{g}$ of protein were loaded on the gels. Protein was measured by the method of Lowry et al. (10) with bovine serum albumin as standard. Estimates of molecular weights of membrane protein aggregates were made by linear extrapolation of plots of log molecular weight vs. mobility curves, with the values assigned to the major membrane proteins by Steck (11) as standards. The polypeptides are numbered $1-8$ according to the convention of Steck (11). The most anodal band is hemoglobin. Two-dimensional separations in polyacrylamide gels were performed essentially as described by Wang and Richards (7). After separation of nonreduced SDS-solubilized membrane proteins in $3 \%$ cylindrical gels, the proteins were then separated in the second dimension in a $5.6 \%$ slab polyacrylamide gel after initial passage through a $2-\mathrm{cm}$ layer of $1 \%$ agarose that contained $10 \%$ mercaptoethanol on top of the slab gel.

Gel filtration chromatography in SDS. Erythrocyte membranes (5-7 $\mathrm{mg}$ protein) were solubilized in $0.01 \mathrm{M}$ Tris buffer, pH 7.8, containing $4 \%$ SDS and $10 \%$ sucrose, layered on a $1 \times 30-\mathrm{cm}$ Sepharose-2B column (Pharmacia Fine Chemicals, Inc., Uppsala, Sweden), and eluted at $25^{\circ} \mathrm{C}$ with $0.01 \mathrm{M}$ Tris buffer, pH 7.8, containing $1 \%$ SDS, at a flow rate of $4 \mathrm{ml} / \mathrm{h}$. In one experiment, $10 \mathrm{mM}$ dithiothreitol was added to the solubilizing solution and the eluting buffer. The absorbance of the eluate was monitored continuously at $280 \mathrm{~nm}$.

Spectrin extraction. The procedure followed was that of Lux et al. (12) with minor modifications. Erythrocyte ghosts were prepared as above, diluted with 1 vol of $1 \mathrm{mM}$ sodium EDTA, $\mathrm{pH} \mathrm{8,} \mathrm{and} \mathrm{dialyzed} \mathrm{against} 1,000 \mathrm{vol}$ of $1 \mathrm{mM}$ sodium EDTA, $\mathrm{pH} 8$, for $48 \mathrm{~h}$ at $4^{\circ} \mathrm{C}$. The supernate and ghost pellet were separated after centrifugation at $30,000 \mathrm{~g}$ for $20 \mathrm{~min}$ and stored overnight at $-20^{\circ} \mathrm{C}$. The total protein content of the ghosts, supernate, and pellets were determined by the method of Lowry et al. (10), and the spectrin content of each fraction was determined by SDS-PAGE, as described above, and scanning in a Gilford densitometer (Model ACD 15, Gilford Instrument Laboratories Inc., Oberlin, Ohio) with an integrating recorder.

Erythrocyte filterability. Filterability of $0.04 \%$ erythrocyte suspensions was determined as described by Palek et al. (13). The data were expressed as the ratio of flow times of erythrocyte suspensions to that of the suspending fluid required for filtration of 1-5 ml of the suspensions.

Separation of erythrocytes by density gradient centrifugation. The $10 \%$ least dense and the $10 \%$ most dense erythrocyte fractions were separated by isopycnic centrifugation in dextran T40 (Pharmacia Fine Chemicals, Inc.) as described by Fitzgibbon et al. (14). $1 \mathrm{ml}$ of washed erythrocytes was layered on $10 \mathrm{ml}$ of $28.5 \%$ ( $w \mathrm{t} / \mathrm{vol}$ ) dextran $\mathrm{T} 40 \mathrm{in} 0.9 \% \mathrm{NaCl}$ (density $=1.109 \mathrm{~g} / \mathrm{ml}$ ) and on $10 \mathrm{ml} 30.5 \%$ dextran $\mathrm{T} 40$ in $0.9 \% \mathrm{NaCl}$ (density $=1.116 \mathrm{~g} / \mathrm{ml})$. After centrifugation in a swinging bucket rotor at $10,000 \mathrm{~g}$ for $1 \mathrm{~h}$, the upper $\cong 10 \%$ erythrocyte fraction which had not entered the $28.5 \%$ dextran and the lower $\cong 10 \%$ erythrocyte fraction which had traversed the $30.5 \%$ dextran solution were collected, washed three times in $0.9 \%$ saline at $4^{\circ} \mathrm{C}$, and membranes prepared and subjected to SDS-PAGE as described above.

Incubations. Erythrocytes were depleted of ATP by incubating $1 \mathrm{vol}$ of washed erythrocytes in $9 \mathrm{vol}$ of glucose-free Dulbecco and Vogt's phosphate-buffered saline (PBS) (15). Penicillin $(100 \mathrm{U} / \mathrm{ml})$ and streptomycin $(0.1 \mathrm{mg} / \mathrm{ml})$ were added to the flasks which were incubated at $37^{\circ} \mathrm{C}$ with shaking at 80 oscillations/min. In some experiments, ATP levels were maintained by addition to the incubation medium of glucose $(20 \mathrm{mM})$ or fructose $(20 \mathrm{mM})$ alone, or glucose $(20 \mathrm{mM})$ together with adenine $(0.5 \mathrm{mM})$ and inosine $(10 \mathrm{mM})$. Periodic $\mathrm{pH}$ adjustments were made during the incubation. After incubation for $24 \mathrm{~h}$, the cells were washed three times with icecold $0.9 \% \mathrm{NaCl}$. One aliquot was taken for the preparation of membranes as above. Additional aliquots were taken for the determination of ATP and reduced glutathione (GSH). ATP was measured in perchloric acid extracts of erythrocytes by the coupled reaction of 3-phosphoglycerate kinase with glyceraldehyde 3-phosphate dehydrogenase as described in leaflets provided by C. F. Boehringer and Sons (Mannheim, West Germany). GSH was measured with the method described by Beutler (16).

GSH stability. Erythrocyte GSH stability to oxidative stress 
with acetylphenylhydrazine was determined according to the conditions of Dacie and Lewis (17).

Light microscopy. 1 vol of heparinized venous blood was immediately fixed with $33 \mathrm{vol}$ of $2 \%$ glutaraldehyde in $310-$ mosmol phosphate buffer. After fixation for $30 \mathrm{~min}$ the cells were observed by phase-contrast microscopy at $\times 1,000$.

\section{RESULTS}

Light microscopy. Phase-contrast microscopy of the GPI-deficient erythrocytes is shown in Fig. 1B. About $50-60 \%$ of the cells were crenated, many of them having spicules. Several cells were cup-shaped. Normal erythrocytes are shown in Fig. 1A.

One-dimensional SDS-PAGE of membranes of nonincubated erythrocytes. SDS-PAGE of freshly prepared nonreduced membranes from GPI-deficient erythrocytes $(51 \%$ reticulocytes) shows striking accumulation of a high molecular weight protein aggregate at the top of the gel (arrowed in Fig. 2, gel e) which completely disappears after mercaptoethanol reduction (Fig. 2, gel f). The position of this aggregate corresponds to an $\cong 700,000 \mathrm{~mol} \mathrm{wt}$, but because the aggregate does not migrate down the gel and complexes could be excluded from the gel, we cannot assign a molecular weight to it. In three preparations, the aggregate comprised between 0.4 and $0.8 \%$ of the total membrane protein as determined by planimetry of the
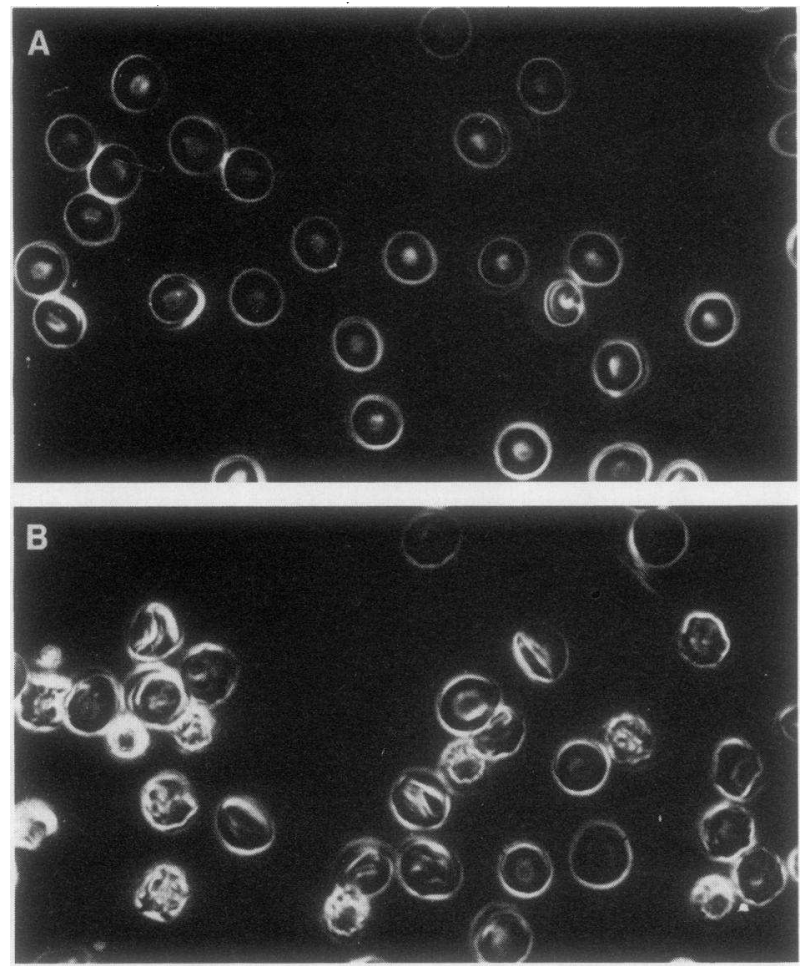

FIGURE 1 Phase photomicrographs of control (A) and GPIdeficient erythrocytes (B).

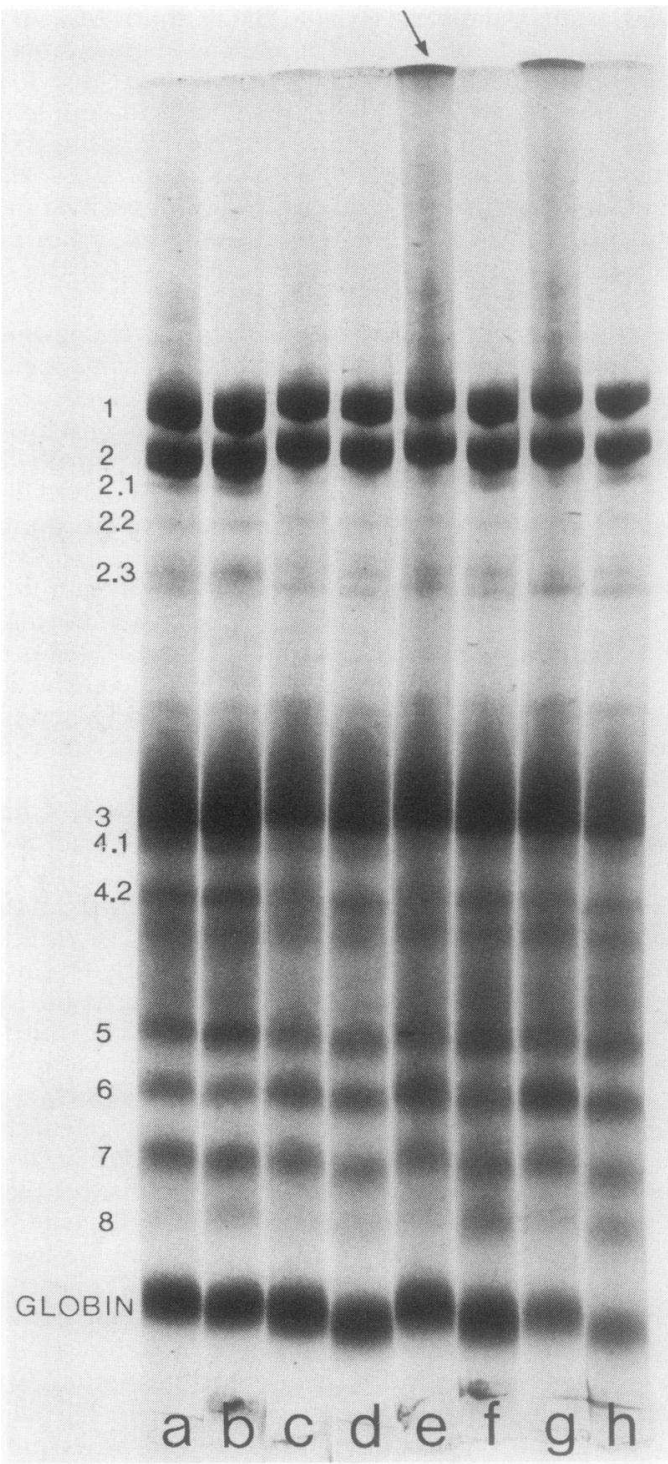

FIGURE 2 SDS-PAGE of erythrocyte membrane proteins. Gels $a$ and $b$, nonreduced and reduced membranes of control erythrocytes; gels $c$ and $d$, nonreduced and reduced membranes of G6PD-deficient erythrocytes (35\% reticulocytes); gels $e$ and $f$, nonreduced and reduced membranes of GPIdeficient erythrocytes (51\% reticulocytes); gels $g$ and $h$, nonreduced and reduced membranes of GPI-deficient erythrocytes incubated for $2 \mathrm{~h}$ in PBS with glucose $(20 \mathrm{mM})$.

gels or by extracting the sliced gel with $66 \%$ (vol/vol) acetic acid and measuring the absorbancy of the extract at $605 \mathrm{~nm}$. Bands 1 and 2 have a reduced staining intensity in the nonreduced preparation $(9.5$ and $11 \%$, respectively, of total membrane protein) when compared with the corresponding values for band 1 and 2 in the reduced preparation of 12.4 and $12.3 \%$, respectively. In addition, the zone between band 1 and the top of the gel in these nonreduced membranes cannot 
be destained as well as in control membranes (Fig. 2, gel a) or in glucose-6-phosphate dehydrogenase (G6PD)-deficient erythrocytes (35\% reticulocytes) (Fig. 2, gel c), neither of which show the high molecular weight aggregate at the top of the gel. Band 8 is prominent in the reduced membrane proteins of the GPI-deficient erythrocytes (Fig. 2, gel f) although it is just visible in the nonreduced preparation (Fig. 2, gel e). The presence and intensity of band 8 is highly variable in control reduced membranes (cf., Fig. 2, gels $b$ and d, and Fig. 3, gels b and d), and we therefore do not regard this as a feature of GPI-deficient erythrocytes.

To exclude the possibility that the high molecular

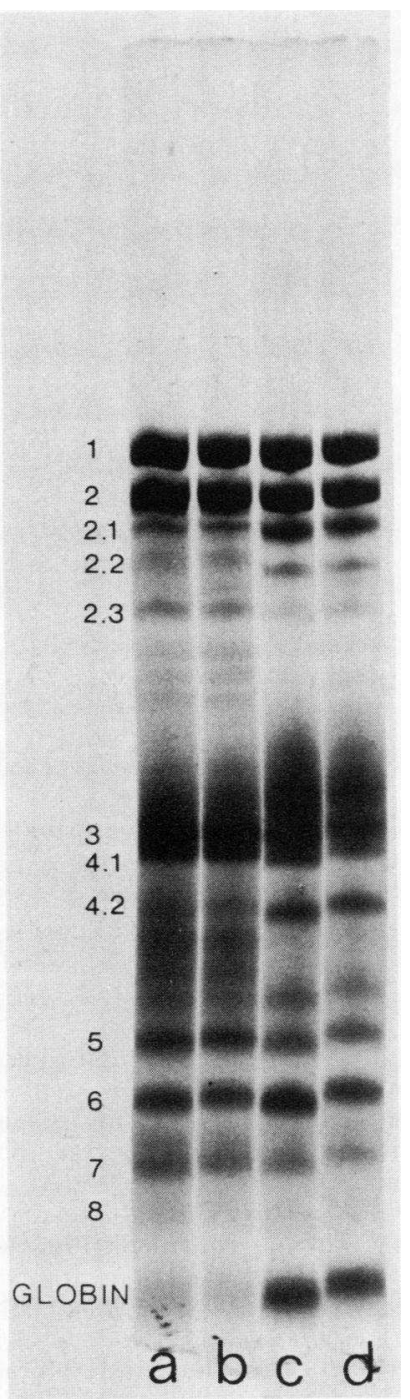

FIGURE 3 SDS-PAGE of erythrocyte membrane proteins. Gels $a$ and $b$, nonreduced and reduced membranes of control erythrocytes; gels $c$ and $d$, nonreduced and reduced membrane proteins of PK-deficient erythrocytes (49\% reticulocytes). weight aggregate might be a feature of erythrocytes or reticulocytes found in splenectomized individuals, nonreduced membrane proteins of a splenectomized patient with pyruvate kinase $(\mathrm{PK})$ deficiency $(49 \%$ reticulocytes) were subjected to SDS-PAGE. Fig. 3 (gel c) shows a complete absence of this aggregate in the PKdeficient erythrocytes when compared with control erythrocytes (Fig. 3, gel a). In addition, we have never seen a high molecular weight aggregate at the top of $3 \%$ gels in numerous preparations of membranes obtained from splenectomized patients with hereditary spherocytosis. The amount of hemoglobin bound by the GPI-deficient erythrocytes is best depicted in Fig. 2 (gel $f$ ) in a reduced membrane preparation, and is slightly increased (14.2\% of total membrane protein) compared with the reduced control or G6PD-deficient membranes (11 and $11.3 \%$ of total membrane protein, respectively [Fig. 2, gels $b$ and d]).

GPI-deficient erythrocytes (41\% reticulocytes) were subjected to density gradient centrifugation in dextran, and the $10 \%$ least dense (62\% reticulocytes) and the $10 \%$ most dense fractions ( $15 \%$ reticulocytes) were collected. SDS-PAGE of the isolated membranes of the two fractions is shown in Fig. 4. The least dense fraction shows complete disappearance of the high molecular weight aggregate at the top of the gel (Fig. 4, gel a), while there is an intensification of this aggregate at the top of the gel in the most dense erythrocyte fraction (Fig. 4, gel b). Membranes from the least dense erythrocyte fraction show almost no binding of hemoglobin in contrast with the marked binding of hemoglobin to the membranes of the most dense erythrocyte fraction.

One-dimensional SDS-PAGE of membranes of incubated erythrocytes. Incubation of the GPI-deficient erythrocytes for $2 \mathrm{~h}$ with glucose $(20 \mathrm{mM})$ has no overt effect on the presence of the high molecular weight aggregate at the top of the gel (Fig. 2, gel g), but does lead to a slight decrease in the amount of membranebound hemoglobin (Fig. 2, gel h). In a separate experiment, incubation of GPI-deficient erythrocytes $(48 \%$ reticulocytes) in a medium containing glucose $(20 \mathrm{mM})$ for a period of $24 \mathrm{~h}$ leads to a marked intensification of the high molecular weight aggregate at the top of the gel in the nonreduced preparation (Fig. 5, gel e). In contrast, control erythrocytes with a normal (1\%) or high reticulocyte count $(46 \%)$ when incubated with glucose do not show this aggregate (Fig. 5 , gels $d$ and f, respectively). The high reticulocyte control preparation was obtained from a patient with autoimmune hemolytic anemia. Addition of adenine $(0.5 \mathrm{mM})$ and inosine $(10 \mathrm{mM})$ to the glucose-containing medium results in almost complete disappearance of the high molecular weight aggregate in the GPI-deficient erythrocytes after $24 \mathrm{~h}$ incubation (Fig. 5 , gel $\mathrm{j}$ ), and to complete disappearance of the aggregate in the low reticulocyte control (Fig. 5, gel i). Incubation of the GPI- 


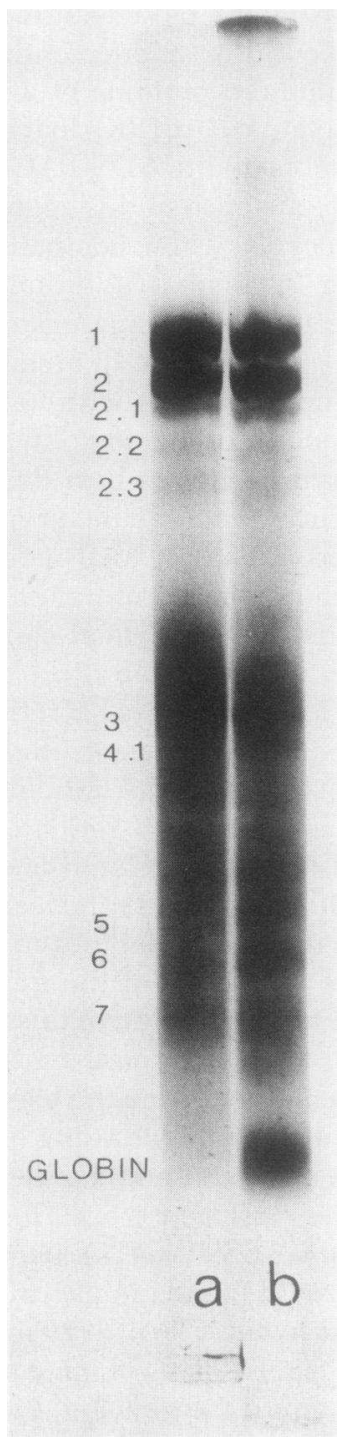

FIGURE 4 SDS-PAGE of GPI-deficient erythrocyte membrane proteins. Gel $a$, nonreduced membranes of the $10 \%$ least dense erythrocyte fraction obtained after density gradient centrifugation; gel $b$, nonreduced membranes of the $10 \%$ most dense erythrocyte fraction.

deficient erythrocytes with fructose $(20 \mathrm{mM})$ as the sole source of substrate also prevents increased aggregate formation (Fig. 5, gel h). Incubation of control erythrocytes (1\% reticulocytes) with fructose for $24 \mathrm{~h}$ completely prevents the formation of the high molecular weight aggregate at the top of the gel (Fig. 5, gel g), but a high molecular weight complex of 450,000 running between band 1 and the top of the gel is seen in this preparation. The latter complex is an artifact as it has not been observed on repeated SDS-PAGE of the

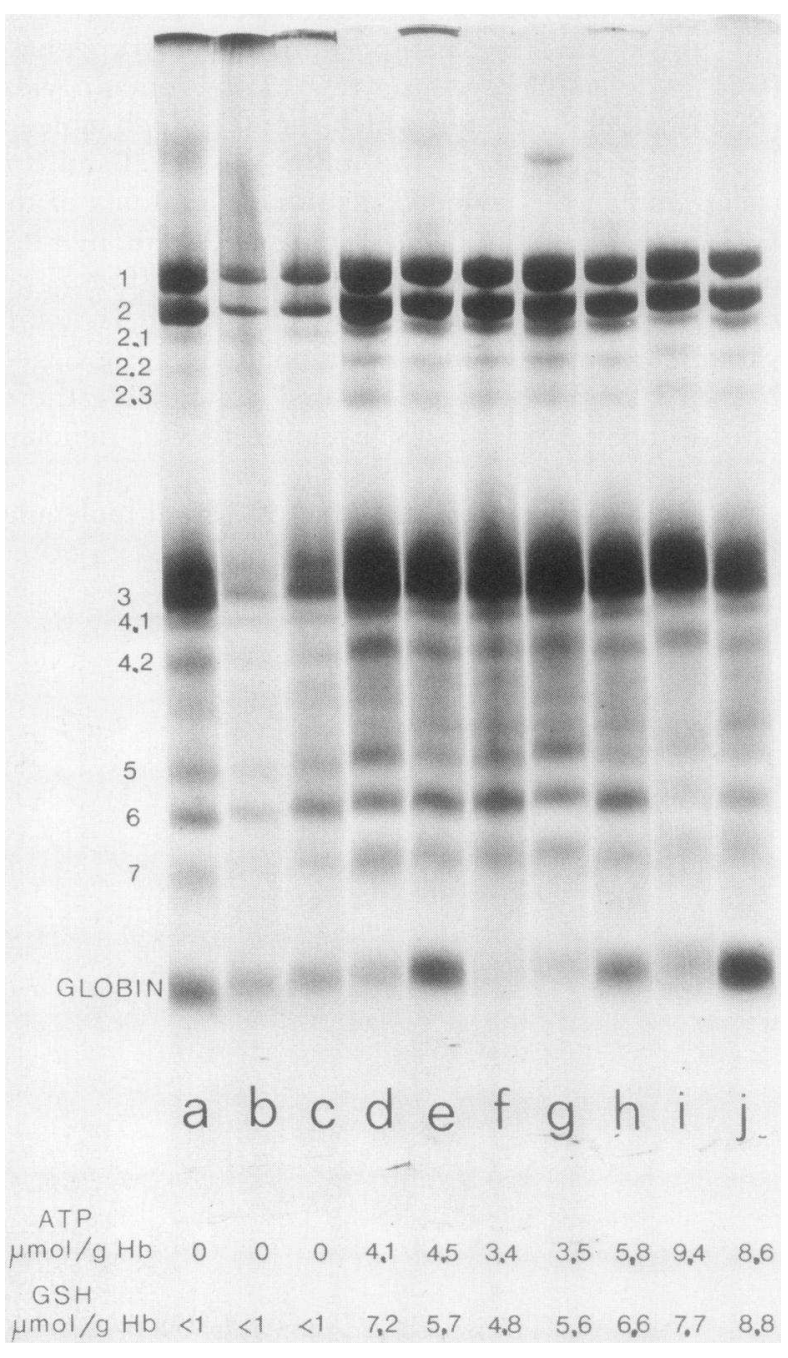

FIGURE 5 SDS-PAGE of nonreduced erythrocyte membrane proteins. Gel $a$, control erythrocytes (1\% reticulocytes) incubated for $24 \mathrm{~h}$ in substrate-free PBS; gel $b$, GPI-deficient erythrocytes (48\% reticulocytes) incubated for $24 \mathrm{~h}$ in substrate-free PBS; gel $c$, control erythrocytes (46\% reticulocytes) incubated for $24 \mathrm{~h}$ in substrate-free PBS; gel $d$, control erythrocytes ( $1 \%$ reticulocytes) incubated for $24 \mathrm{~h}$ in PBS that contained glucose $(20 \mathrm{mM})$; gel $e$, GPI-deficient erythrocytes incubated for $24 \mathrm{~h}$ in PBS that contained glucose $(20 \mathrm{mM})$; gel $f$, control erythrocytes (46\% reticulocytes) incubated for $24 \mathrm{~h}$ in PBS that contained glucose $(20 \mathrm{mM})$; gel $g$, control erythrocytes (1\% reticulocytes) incubated for $24 \mathrm{~h}$ in PBS that contained fructose $(20 \mathrm{mM})$; gel $h$, GPI-deficient erythrocytes incubated for $24 \mathrm{~h}$ in PBS that contained fructose $(20 \mathrm{mM})$; gel $i$, control erythrocytes ( $1 \%$ reticulocytes) incubated for $24 \mathrm{~h}$ in PBS that contained glucose $(20 \mathrm{mM})$, adenine $(0.5 \mathrm{mM})$, and inosine ( $10 \mathrm{mM})$; gel $j$, GPI-deficient erythrocytes incubated for $24 \mathrm{~h}$ in PBS that contained glucose $(20 \mathrm{mM})$, adenine $(0.5$ $\mathrm{mM})$, and inosine $(10 \mathrm{mM})$. ATP and GSH levels after incubation under the various conditions are shown below the corresponding gels. 
same membrane preparation and in two other control erythrocyte preparations incubated with fructose.

ATP and GSH levels of the GPI-deficient erythrocytes and low and high reticulocyte controls incubated under the various conditions above are shown as subscripts to the relevant gels in Fig. 5. In the GPIdeficient erythrocytes incubated with glucose, ATP levels are slightly higher than in the low and high reticulocyte controls incubated with glucose. GPIdeficient erythrocytes incubated with fructose show an even higher level of ATP. Addition of adenine and inosine to the glucose-containing medium results in an approximate doubling of ATP levels in both the GPIdeficient erythrocytes and the low reticulocyte controls, presumably as a result of replenishment of the adenine nucleotide pool. In the absence of substrate, ATP is undetectable in all incubations. GSH levels in the GPI-deficient erythrocytes incubated with glucose are intermediate between the high and low reticulocyte controls. In the GPI-deficient cells incubated with fructose GSH levels are slightly higher than in the low reticulocyte control. Highest values of GSH are found in the control and GPI-deficient cells incubated in a glucose-containing medium replenished with adenine and inosine. Preincubation levels of ATP in the low reticulocyte control, the GPI-deficient cells, and the high reticulocyte control were $4.4,4.7$, and $4.4 \mu \mathrm{mol} / \mathrm{g}$ hemoglobin, respectively. The corresponding preincubation levels of GSH were 7.5, 8.0 and $8.4 \mu \mathrm{mol} / \mathrm{g}$ hemoglobin, respectively.

Incubation for $24 \mathrm{~h}$ without substrate leads to the development of an intensely staining high molecular weight aggregate at the top of the gels in membranes of the GPI-deficient (Fig. 5, gel b) and control erythrocytes with normal reticulocyte count (Fig. 5, gel a) and high reticulocyte count (46\%) (Fig. 4, gel c). Although both the GPI-deficient reticulocytes and the high reticulocyte control show less residual spectrin, band $3,4.1,4.2$, and 5 , than the low reticulocyte control, the effect is even more pronounced in the GPIdeficient erythrocytes.

Two-dimensional SDS-PAGE of erythrocyte membranes. Two-dimensional SDS-PAGE of membranes of GPI-deficient erythrocytes with reduction to cleave disulphide bonds before separation in the second dimension is shown in Fig. 6A. The intermediate agarose gel which contained $10 \%$ mercaptoethanol is not shown. The individual components on the slab gel are numbered and fall in a diagonal skewed line. The offdiagonal spots in the area of bands $3-5$, which stain poorly, represent glycoproteins. Membrane protein aggregates arising from disulphide bonding are also seen as off-diagonal spots at the same vertical position of the original membrane aggregate in the first-dimensional separation. These consist primarily of band 1 in the region extending from band 1 to the high molecular weight aggregate at the top of the first dimension gel with only traces of band 2, and also a spot (solid arrow) corresponding to 4.2 in the vertical line of the high molecular weight aggregate at the top of the gel. In addition, a faint spot (dotted arrow) corresponding to band 8 is seen at the vertical position of band 6 , and probably represents a dimer of band 8 comigrating with band 6 in the first-dimension gel. These findings are contrasted with the more extensive cross-linking found on two-dimensional SDS-PAGE of membrane proteins in ATP-depleted erythrocytes obtained after $24 \mathrm{~h}$ incubation in substrate-free PBS (Fig. 6B). Bands 1 and 2 form the major components of the high molecular weight aggregate at the top of the one-dimensional gel, although most other membrane proteins are also involved. There is, in addition, an aggregate of apparent $\cong 400,000$ mol wt (a spectrin heterodimer) and a complex of apparent $250,000 \mathrm{~mol}$ wt, which consists of either band 1 or 2 , and a small molecular weight protein which is not visible in this gel. Band 8 does not appear to comigrate with band 6 in the first-dimension separation, ${ }^{2}$ as found in the GPI-deficient erythrocyte.

Gel-filtration chromatography. As it was possible that membrane protein complexes $>700,000 \mathrm{~mol} w \mathrm{wt}$ might be excluded from $3 \%$ polyacrylamide gels, membrane proteins were dissolved in SDS and subjected to chromatography on Sepharose-2B. Membrane proteins of ATP-depleted erythrocytes (24-h incubation in PBS) show a high molecular weight aggregate $\left(>40 \times 10^{6}\right)$ at the void volume of the column (Fig. 7B) which completely disappears after reduction with dithiothreitol (not shown). Freshly prepared nonreduced membranes from normal adult erythrocytes do not show this peak (Fig. 7A), but a similar preparation of the GPI-deficient erythrocytes shows a small peak $(0.3 \%$ of total membrane proteins) at the void volume (Fig. 7C), which does not show any alteration after reduction with dithiothreitol (Fig. 7D).

Spectrin extractability. In accordance with the findings of Lux et al. (12), extraction of erythrocyte membranes with $1 \mathrm{mM}$ EDTA, $\mathrm{pH} 8.0$, resulted in the selective solubilization of spectrin, actin, and traces of hemoglobin. 68 and $69 \%$ of spectrin was extracted from membranes of fresh erythrocytes obtained from two normal controls. By contrast, only $36 \%$ of spectrin was extracted from membranes of fresh GPI-deficient erythrocytes.

\footnotetext{
${ }^{2}$ Palek et al. (18) have recently found that membranes of ATP-depleted erythrocytes contain a 260,000-mol wt complex which is a heterodimer of band 2 and 4.9 , and that band 8 and part of band 5 comigrate with band 6 in nonreduced membranes of such erythrocytes.
} 

12
$3 \quad 4.2$
$5 \quad 6 \quad 7$
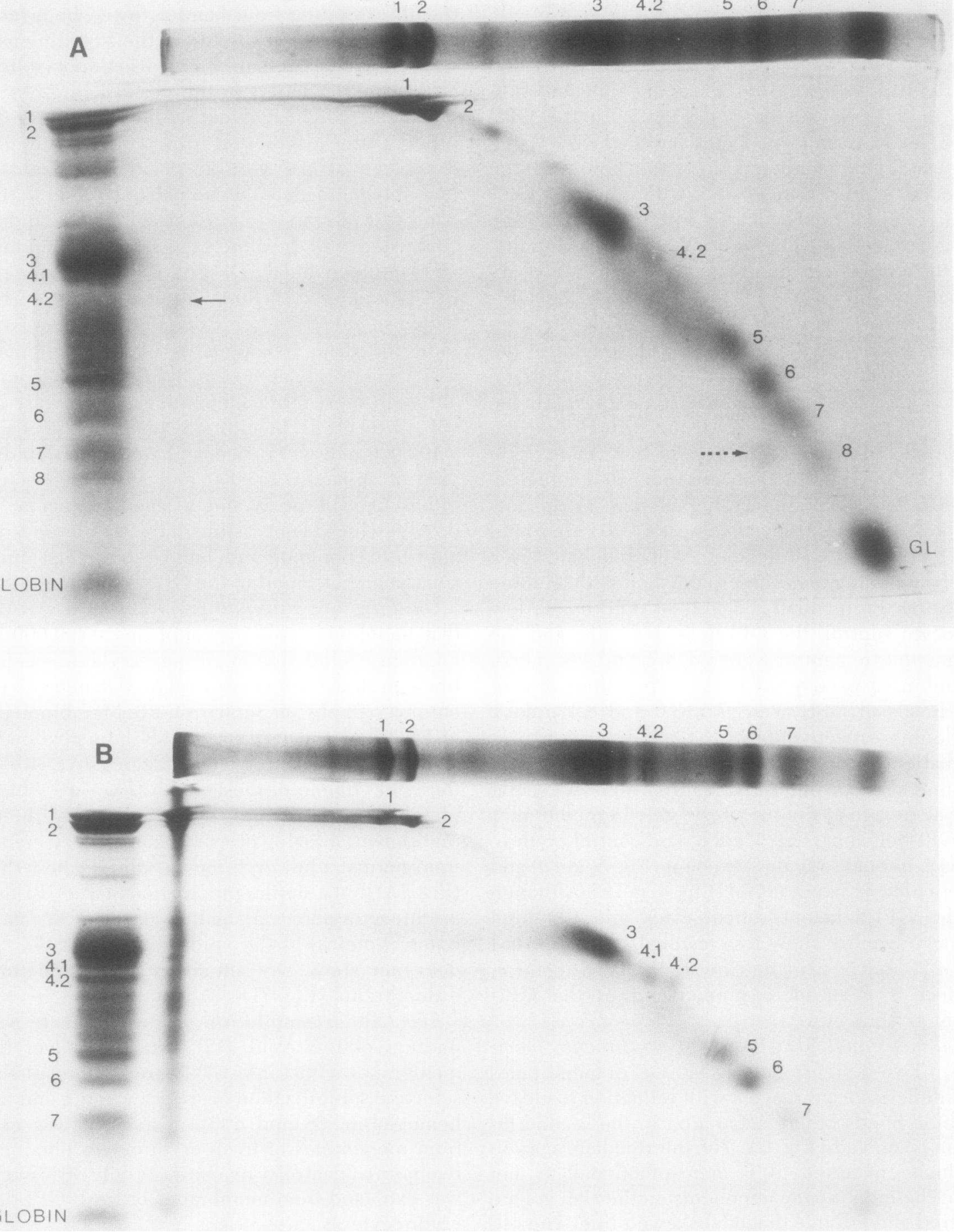

FigURE 6 Two-dimensional SDS-PAGE of erythrocyte membrane proteins. (A), membranes of freshly prepared GPI-deficient erythrocytes. (B), membranes of control erythrocytes incubated for $24 \mathrm{~h}$ in substrate-free PBS. Control membranes are shown at the left of the slab gels as membrane protein markers. The first-dimension gels are shown lying horizontally on the top of the slab gels. The agarose layer that contained $10 \%$ mercaptoethanol through which the membrane proteins are electrophoresed after separation on the first dimension is not shown. 


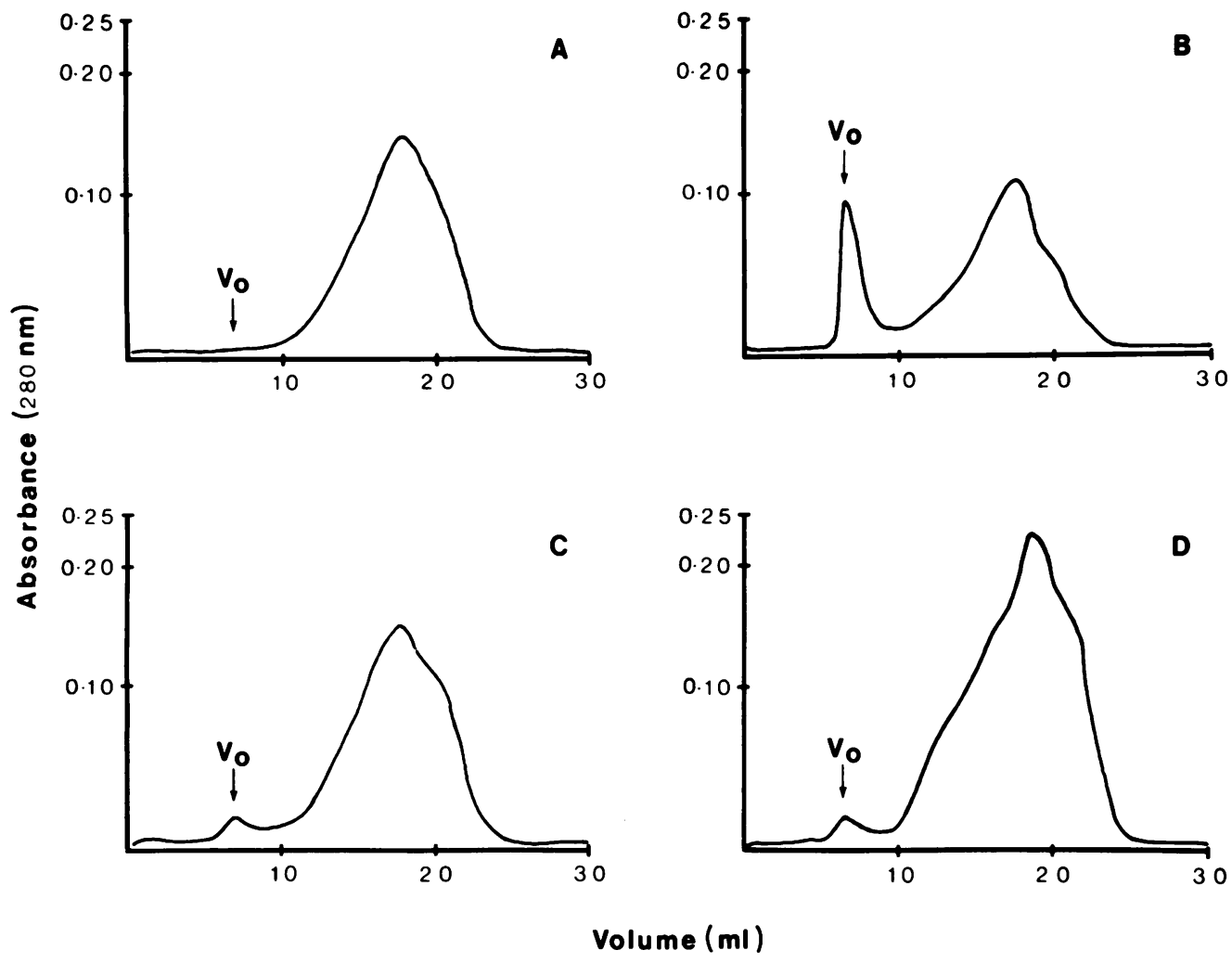

FIGURE 7 Gel filtration chromatography of SDS-solubilized membrane proteins. (A), control nonreduced membranes; (B), nonreduced membranes of control erythrocytes incubated for $24 \mathrm{~h}$ in PBS (no glucose); (C), nonreduced membranes of GPI-deficient erythrocytes; (D), reduced membranes of GPI-deficient erythrocytes.

Erythrocyte filterability. The ratio of the flow times of $1-5 \mathrm{ml}$ of $0.04 \%$ erythrocyte suspensions from three controls and the patient with GPI-deficiency to that of the suspending medium ( $\mathrm{pH}$ 7.4) used for filtration is shown in Fig. 8. This ratio is higher for GPI-deficient erythrocytes, particularly at 3-, 4-, and 5 -ml vol, indicating that filterability of these erythrocytes is impaired.

GSH stability. Reduced GSH levels in fresh unincubated GPI-deficient erythrocytes (40\% reticulocytes) was $6.8 \mu \mathrm{mol} / \mathrm{g}$ hemoglobin (normal range $6.6 \pm 1.0$ $\mu \mathrm{mol} / \mathrm{g}$ hemoglobin). There was a $15 \%$ fall on incubation of the erythrocytes with acetylphenylhydrazine (normal response: $<20 \%$ fall in GSH levels).

\section{DISCUSSION}

The striking feature of this study of erythrocyte membrane proteins in a splenectomized patient with a thermolabile variant of GPI-deficiency is the presence of a high molecular weight aggregate of membrane proteins on SDS-PAGE, which completely disappears on reduction with mercaptoethanol. This aggregate,

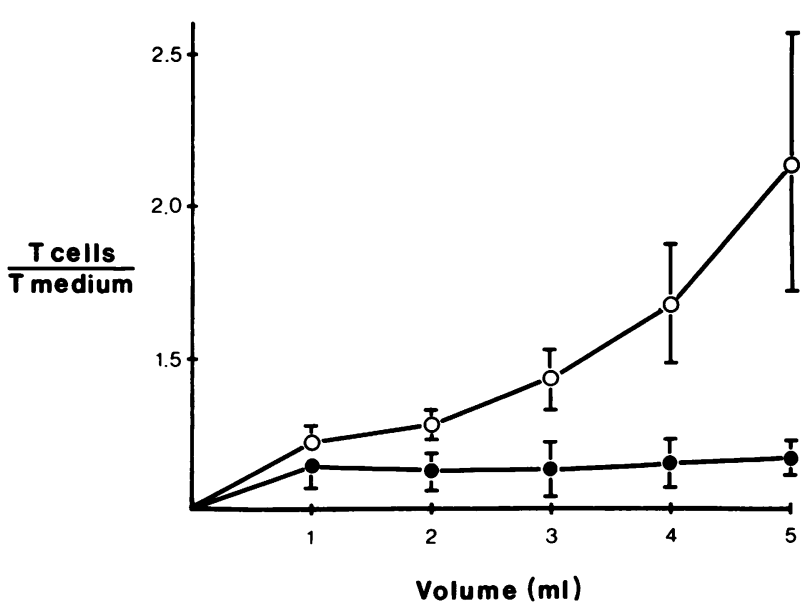

FIGURE 8 Filterability of control and GPI-deficient erythrocytes. The ratio of the flow time of $0.04 \%$ erythrocyte suspensions to the flow time of the suspending medium is plotted against volume. For the controls (closed circles) each point represents the mean $\pm S D$ of three observations on three subjects (nine observations), and for the GPI-deficient erythrocytes (open circles) each point represents the mean $\pm \mathrm{SD}$ of four observations. 
which represents $0.4-0.8 \%$ of total membrane protein, is not detectable in the membranes of a splenectomized child with PK deficiency with a comparable reticulocyte count or in a patient with G6PD-deficiency and $35 \%$ reticulocytosis.

Allen et al. (19) have recently described a patient with a hemolytic anemia associated with G-6-PD, Long Prairie, in whom mercaptoethanol-reducible high molecular weight aggregates of $4.4 \times 10^{5}$ and $>50$ $\times 10^{6}$ were found in the isolated erythrocyte membranes. Our finding of mercaptoethanol-reducible aggregates in the membranes of the GPI-deficient erythrocytes is thus not specific for these erythrocytes. The molecular weight of the membrane protein aggregate is at least $7 \times 10^{5}$ as determined by its position at the top of $3 \%$ polyacrylamide gels (Figs. 2 and 6A). However, we cannot assign a molecular weight to it as the aggregate does not migrate down the gel. An aggregate of membrane proteins $(30 \%$ of total membrane protein) is also seen in the same position in normal erythrocytes which have been ATPdeleted (Fig. 6B) which others (18) have found to be a result of disulphide-bonding of membrane proteins. The latter aggregate has a molecular weight of $>40$ $\times 10^{6}$ (Fig. 7B) which disappears completely on reduction with mercaptoethanol. The membranes of the GPIdeficient erythrocytes also show a small peak of molecular weight $>40 \times 10^{6}$ but this is stable to mercaptoethanol reduction. This peak represents the nondisulphide-bonded high molecular weight aggregate found in membranes of erythrocytes of splenectomized subjects which had recently been described by Lux and John (20). Because this complex is quantitatively similar in both reduced and non-reduced preparations in the GPI-deficient erythrocytes, the high molecular weight aggregate at the top of the first dimension $3 \%$ polyacrylamide gels in nonreduced membranes is probably present in the inclusion volume of the Sepharose2B column, i.e., <mol wt $40 \times 10^{6}$, although we cannot be certain of this as it forms a relatively small proportion of the total membrane proteins of GPIdeficient erythrocytes.

The membrane protein aggregate of GPI-deficient erythrocytes appears to consist primarily of band 1 and a band with the mobility of 4.2 , and differs in this respect from ATP-depleted erythrocytes in which both band 1 and band 2 form the major constituents of the high molecular weight aggregate, with lesser amounts of several other membrane proteins. Attempts to increase the amount of membrane protein of GPIdeficient erythrocytes applied to the first-dimension gel led to poorer resolution in the second dimension; therefore we cannot exclude the possibility that other membrane proteins are involved in the high molecular weight aggregate of these erythrocytes. After resolution in the second-dimension gel, band 8 was found to run in the vertical position of band 6 and probably exists as a dimer in the nonreduced membranes. An identical observation was made by Palek et al. (18) for ATP-depleted erythrocytes, although we were unable to detect this in our own experiments (Fig. 6B). ATP-depleted cells also show a complex of $\cong \mathrm{mol} \mathrm{wt}$ 250,000 which can be seen just cathodal to band 1 (Fig. $6 \mathrm{~B}$ ), and which was found by Palek et al. (18) to consist of band 2 plus 4.9. This complex, which was thought to be a result of concomitant depletion of reduced GSH in erythrocytes undergoing ATP-depletion, was not present in the freshly prepared membranes of the GPIdeficient erythrocytes. This is consistent with the normal levels of reduced GSH and normal GSH stability which were found in this patient and which were not previously reported.

An interesting feature of the GPI-deficient erythrocytes is the marked decrease in spectrin extractability of freshly prepared membranes. This is similar to that found by Lux et al. (12) in ATP-depleted erythrocytes. One of the hallmarks of such ATPdepleted erythrocytes is a decrease in their apparent membrane deformability, a property which was also a striking feature of the GPI-deficient erythrocytes (Fig. 8), insofar as filterability is a measure of erythrocyte deformability. It is possible that the surface irregularity of the crenated cells could contribute to the decreased deformability, but the degree to which it does so is unknown.

A finding of considerable interest in this study was the inability of glucose to prevent the development of increased amounts of the high molecular weight aggregate in GPI-deficient erythrocytes incubated for 24 $h$ in PBS, whereas bypassing the glycolytic defect by the addition of adenine and inosine or the use of fructose as the sole substrate prevented this phenomenon (Fig. 5). This suggests that these erythrocytes might not be able to generate sufficient ATP or some other metabolite distal to the block in glycolysis which is necessary to prevent aggregate formation. However, ATP (and GSH) levels were, in fact, not depressed and were similar to that found in the low and high reticulocyte controls. These findings are difficult to explain, particularly if ATP depletion is indeed etiologically related to the development of disulphide-bonded membrane aggregates and decreased extractability of spectrin in these erythrocytes, as found by others $(12,18)$ for normal erythrocytes undergoing ATP depletion. Our findings, however, must also be reconciled with the possibility that susceptibility to aggregate formation could be a function of only a small pool of older or nearly effete erythrocytes, as suggested by our finding that in GPI-deficient erythrocytes aggregates are found in the oldest (most dense) erythrocyte fraction and are completely absent in the youngest (least dense) fraction. As we have sampled only the 
whole cell population in the incubation experiments, the findings of normal ATP and GSH levels in the GPI-deficient erythrocytes may mask a more severe metabolic defect in a small population of older cells. If this hypothesis is correct, then bypassing the defect with either adenine and inosine or fructose prevents aggregate formation in only a small pool of susceptible cells. Although we have limited ourselves to attempting to correlate ATP and GSH levels with aggregate formation in these experiments, other metabolites may be of equal or greater importance in defining such a relationship.

A further point of interest relates to the binding of hemoglobin to the membranes of GPI-deficient erythrocytes. Schröter and Tillman (3) found a highly significant increase in the amount of hemoglobin bound to the erythrocyte membranes of four subjects with GPI deficiency, which they correlated with the apparent decrease in deformability of these erythrocytes. Although binding of hemoglobin to the erythrocyte membranes of the patient described in this study was only slightly increased compared with a low reticulocyte control, there was a very marked increase in the amount of hemoglobin bound to the membranes of the $10 \%$ most dense fraction, while almost no hemoglobin was bound to the $10 \%$ least dense or youngest cell fraction. The relationship of this finding to possible alteration of the membrane properties of these cells requires further study.

Although the susceptibility to develop aggregates appears to be directly related to an impairment of substrate flow through the glycolytic sequence, the exact mechanism of aggregate formation in these GPIdeficient erythrocytes remains to be elucidated. However, the finding of membrane aggregates probably linked by disulphide bonds and involving spectrin, as well as the decreased extractability of spectrin from these membranes, make it tempting to speculate on possible mechanisms of hemolysis in the GPI-deficient erythrocytes studied by us. In accordance with the concepts of Lux et al. (12), it is possible that there is a change in the physical state of spectrin in these membranes, which affects its interaction with integral membrane proteins or lipids, leading to decreased membrane deformability and splenic or liver sequestration.

\section{ACKNOWLEDGMENTS}

These studies were supported by grants from the South African Medical Research Council and the Atomic Energy Board.

\section{REFERENCES}

1. Paglia, D. E., and W. N. Valentine. 1974. Hereditary glucosephosphate isomerase deficiency. A review. Am.J. Clin. Pathol. 62: 740-751.

2. Schröter, W., and W. Tillman. 1977. Congenital non- spherocytic hemolytic anemia associated with glucosephosphate isomerase deficiency: Variant Paderborn. Klin. Wochenschr. 55: 393-396.

3. Schröter, W., and W. Tillman. 1977. Decreased deformability of erythrocytes in haemolytic anaemia associated with glucosephosphate isomerase deficiency. $\mathrm{Br} . \mathrm{J}$. Haematol. 36: 475-484.

4. Cayanis, E., G. K. Penfold, I. Freiman, and L. G. MacDougall. 1977. Haemolytic anaemia associated with glucosephosphate isomerase deficiency in a black South African child. Br. J. Haematol. 37: 363-371.

5. Oski, F. A., and J. L. Naiman. 1965. Red cell metabolism in the premature infant. I. Adenosine triphosphate levels, adenosine triphosphate stability, and glucose consumption. Pediatrics. 36: 104-112.

6. Steck, T. L. 1972. Cross-linking the major proteins of the isolated erythrocyte membrane. J. Mol. Biol. 66: 295-305.

7. Wang, K., and F. M. Richards. 1974. An approach to nearest neighbour analysis of membrane proteins. Application to the human erythrocyte membrane of a method employing cleavable cross-linkages. J. Biol. Chem. 249: 8005-8008.

8. Liu, S. C., G. Fairbanks, and J. Palek. 1977. Spontaneous. reversible protein cross-linking in the human erythrocyte membrane. Temperature and $\mathrm{pH}$ dependence. Biochemistry. 16: 4066-4074.

9. Fairbanks, G., T. L. Steck, and D. F. H. Wallach. 1971. Electrophoretic analysis of the major polypeptides of the human erythrocyte membrane. Biochemistry. 10: 26062617.

10. Lowry, O. H., N. J. Rosebrough, A. L. Farr, and R. J. Randall. 1951. Protein measurement with the Folin phenol reagent. J. Biol. Chem. 193: 265-275.

11. Steck, T. L. 1974. The organization of proteins in the human red blood cell membrane. A review. J. Cell Biol. 62: $1-19$.

12. Lux, S. E., K. M. John, and T. E. Ukena. 1978. Diminished spectrin extraction from ATP-depleted human erythrocytes. Evidence relating spectrin to changes in erythrocyte shape and deformability. J. Clin. Invest. 61: 815-827.

13. Palek, J., A. Liu, D. Liu, M. Snyder, N. L. Fortier, G. Njoku, F. Kiernan, D. Furk, and T. Crasberg. 1977. Effect of procaine $\mathrm{HCl}$ on ATP: calcium-dependent alterations in red cell shape and deformability. Blood. 50: 155- 164.

14. Fitzgibbon, J. F., R. D. Koler, and R. T. Jones. 1976. Red cell age-related changes of hemoglobins $A_{I a+b}$ and $A_{I c}$ in normal and diabetic subjects. J. Clin. Invest. 58: 820-824.

15. Dulbecco, R., and M. Vogt. 1954. Plaque formation and isolation of pure lines with poliomyelitis viruses. J. Exp. Med. 99: 167-182.

16. Beutler, E. 1975. Red Cell Metabolism. A Manual of Biochemical Techniques. Grune \& Stratton, Inc., New York. 2nd edition. 112-114.

17. Dacie, J. V., and S. M. Lewis. 1963. Practical Haematology. J. and A. Churchill Ltd., London. 3rd edition. 393.

18. Palek, J., S. C. Liu, and L. M. Snyder. 1978. Metabolic dependence of protein arrangement in human erythrocyte membranes. I. Analysis of spectrin rich complexes in ATP-depleted red cells. Blood. 51: 385-395.

19. Allen, D. W., G. J. Johnson, S. Cadman, and M. E. Kaplan. 1978. Membrane polypeptide aggregates in glucose-6phosphate dehydrogenase-deficient and in vitro aged red blood cells. J. Lab. Clin. Med. 91: 321-327.

20. Lux, S. E., and K. M. John. 1977. Isolation and partial characterisation of a high molecular weight red cell membrane protein complex normally removed by the spleen. Blood. 50: 625-645. 